\title{
Dipolar cortico-muscular electrical stimulation: a novel method that enhances motor function in both - normal and spinal cord injured mice
}

\author{
Zaghloul Ahmed
}

\begin{abstract}
Background: Electrical stimulation of the central and peripheral nervous systems is a common tool that is used to improve functional recovery after neuronal injury.

Methods: Here we described a new configuration of electrical stimulation as it was tested in anesthetized control and spinal cord injury (SCl) mice. Constant voltage output was delivered through two electrodes. While the negative voltage output (ranging from -1.8 to $-2.6 \mathrm{~V}$ ) was delivered to the muscle via transverse wire electrodes (diameter, $500 \mu \mathrm{m}$ ) located at opposite ends of the muscle, the positive output (ranging from +2.4 to $+3.2 \mathrm{~V}$ ) was delivered to the primary motor cortex (M1) (electrode tip, $100 \mu \mathrm{m}$ ). The configuration was named dipolar corticomuscular stimulation (dCMS) and consisted of 100 pulses (1 ms pulse duration, $1 \mathrm{~Hz}$ frequency).

Results: In SCI animals, after dCMS, cortically-elicited muscle contraction improved markedly at the contralateral (456\%) and ipsilateral (457\%) gastrocnemius muscles. The improvement persisted for the duration of the experiment $(60 \mathrm{~min})$. The enhancement of cortically-elicited muscle contraction was accompanied by the reduction of M1 maximal threshold and the potentiation of spinal motoneuronal evoked responses at the contralateral (313\%) and ipsilateral (292\%) sides of the spinal cord. Moreover, spontaneous activity recorded from single spinal motoneurons was substantially increased contralaterally (121\%) and ipsilaterally (54\%). Interestingly, spinal motoneuronal responses and muscle twitches evoked by the test stimulation of non-treated M1 (received no dCMS) were significantly enhanced as well. Similar results obtained from normal animals albeit the changes were relatively smaller.
\end{abstract}

Conclusion: These findings demonstrated that dCMS could improve functionality of corticomotoneuronal pathway and thus it may have therapeutic potential.

\section{Introduction}

After a spinal cord injury (SCI), spared regions of the central nervous system are spontaneously capable of repairing the damaged pathway, although the process is very limited. Moreover, despite the many promising treatment strategies to improve connections across the damaged spinal cord, the strength of connectivity and functional recovery of the impaired spinal cord is still unsatisfactory. It is well known that spared axons sprout after an SCI [1-3], but fine-tuning of this process as well as synapse stabilization might be dependent on precise

Correspondence: zahmed6701@gmail.com

Department of Physical Therapy and Neuroscience Program, The College of Staten Island/CUNY, 2800 Victory Boulevard, Staten Island, NY 10314, USA pathway-selective activity. Electrical stimulation is an effective method that promotes reactive sprouting through which an increase in the number of functional connections may be possible [3]. Electrical stimulation can also improve functional connections by strengthening the weak existing synapses and/or by promoting synaptogenesis. Of relevance, one of the emerging concepts is that the nervous system contains latent pathways that can be awoken by electrical stimulation or pharmacological manipulation [3-8].

The majority of the methods employing electrical stimulation use unipolar or bipolar stimuli delivered locally at one region of the nervous system. The loss of neuromuscular activity after SCI leads to inevitable abnormalities that limit the effectiveness of localized stimulation. 
Some of these abnormalities are muscle atrophy [9-12] and peripheral nerve inexcitability $[13,14]$. Furthermore, changes of the sensorimotor pathway below and above the lesion may involve several different mechanisms; some of them may be maladaptative [15-17]. This maladaptive function will bias stimuli toward connections with better integrity, further limiting the effectiveness of localized stimulation.

According to the Habbian plasticity principle [18], physiological processes strengthen synaptic connections when presynaptic activity correlates with postsynaptic firing. This phenomenon is known as long term potentiation (LTP) [19]. LTP could be induced by high-frequency presynaptic stimulation or by pairing lowfrequency stimulation with postsynaptic depolarization. LTP can also be induced if a pre-synaptic input is activated concurrently with post-synaptic input [20]. In addition, direct current passed through a neuronal pathway can modulate the excitability of that pathway depending on the current polarity and neuronal geometry $[21,22]$. In that, anodal stimulation would excite while cathodal stimulation inhibits neuronal activity. Drawing from these principles and findings, it was predicted in the present study that encompassing characteristics of current application like pairing cortical with muscular stimulation combined with polarizing current would initiate physiological processes that strengthen connections of the corticomotoneuronal pathways weakened by SCI.

In the present study, we asked the question whether the passage of pulsed direct current across the corticomotoneuronal pathway promotes stronger connections between spinal motor circuits and the motor cortex. Given the electrodes' location, this configuration was called dipolar cortico-muscular stimulation (dCMS). The positive electrode was situated at the motor cortex and the negative electrode was at the contralateral partially isolated gastrocnemius muscle. Here, it was demonstrated that dCMS substantially improved cortically-elicited muscle contractions and spinal cord responses in control and SCI animals.

\section{Methods}

\section{Animals}

Experiments were carried out on CD-1, male and female adult mice in accordance with $\mathrm{NIH}$ guidelines. All protocols were approved by the College of Staten Island IACUC. Animals were housed under a $12 \mathrm{~h}$ light-dark cycle with free access to food and water.

\section{Spinal cord contusion injury}

Mice were deeply anaesthetized with ketamine/xylazine (90/10 mg/kg i.p.). A spinal contusion lesion was produced ( $\mathrm{n}=15$ mice) at spinal segment T13 using the
MASCIS/NYU impactor [23]. $1 \mathrm{~mm}$-diameter impact head $\operatorname{rod}(5.6 \mathrm{~g})$ was released from a distance of 6.25 mm onto T13 spinal cord level exposed by a T10 laminectomy. After injury, the overlying muscle and skin was sutured, and the animals were allowed to recover under a heating lamp at $30^{\circ} \mathrm{C}$. To prevent infection after the wound was sutured, a layer of ointment contained gentamicin sulfate was applied. Following surgery, animals were maintained under pre-operative conditions for 120 days before testing. The time of recovery was selected to ensure that animals developed a stable chronic spinal cord injury.

\section{Behavioral testing}

Behavioral testing ( $\mathrm{n}=15$ animals with $\mathrm{SCI}$ ) was performed 120 days post-injury to confirm that animals developed behavioral signs of locomotor abnormalities, spasticity syndrome, and sensorimotor incoordination at the hindlimbs. We have only used animals that demonstrated higher (approximately symmetrical in both hindlimbs) behavioral abnormalities. After acclimatization to the test environment, three different testing procedures were used to quantify these behavioral problems.

\section{Basso mouse scale (BMS)}

Motor ability of the hindlimbs was assessed by the motor rating of BMS [24]. The rating is as follows: 0 , no ankle movement; $1-2$, slight or extensive ankle movement; 3 , planter placing or dorsal stepping; 4 , occasional planter stepping; 5 , frequent or consistent planter stepping; no animal scored more than 5. Each mouse was observed for $4 \mathrm{~min}$ in an open space, before a score was given.

\section{Abnormal pattern scale (APS)}

After SCI, animals usually developed muscle tone abnormalities that were exaggerated during locomotion and lifting the animal off the ground (by the tail). We developed APS to quantify the number of muscle tone abnormalities demonstrated by animals after SCI in two situations: on ground and off ground. The rating is as follows: 0 , no abnormalities; 1 , for each of the following abnormalities: limb crossing of midline, abduction, and extension or flexion of the hip joint, paws curling or fanning, knee flexion or extension, ankle dorsi or planter flexion. The total score is the sum of abnormalities from both hindlimbs. The maximal score in APS is 12 . Abnormal patterns were usually accompanied by spasmodic movements of the hindlimbs.

\section{Horizontal ladder scale (HLS)}

For accurate placing for the hindlimb, animals have to have control coordination between sensory and motor systems. To test for sensorimotor coordination, we used a grid with equal spacing $(2.5 \mathrm{~cm})$. Animals were placed on the grid and were allowed to take 20 consecutive steps. Foot slips were counted as errors. 


\section{Electrophysiological procedures}

Intact $(\mathrm{n}=10)$ and SCI $(\mathrm{n}=21)$ animals underwent a terminal electrophysiological experiment. Animals were anesthetized using ketamine/xylazine (90/10 mg/kg i.p.), which was found to preserve corticospinal evoked potential [3,25-27]. Electrophysiological procedures started approximately $45 \mathrm{~min}$ after the first injection to maintain anesthesia at light to moderate level, as recommended by Zandieh and colleagues [25]. Anesthesia was kept at this level using supplemental dosages ( $5 \%$ of the original dose).

The entire dorsal side of each animal was shaved. The skin covering the two hindlimbs, lumbar spine, and the skull was removed. Both gastrocnemii muscles were carefully separated from the surrounded tissue preserving blood supply and nerves. The tendon of each of the muscles was threaded with a hook shaped 0-3 surgical silk, which was connected to the force transducers. Next, we performed a laminectomy in the $2^{\text {nd }}, 3^{\text {rd }}$, and $4^{\text {th }}$ lumbar vertebrae (below the lesion in animals with $\mathrm{SCI})$; the $13^{\text {th }}$ rib was used as a bone land mark to identify the level of spinal column. Since spinal cord levels are $\sim 3$ level displaced upward relative to vertebral levels, we assumed that recording was performed at spinal cord levels: $5^{\text {th }}$ and $6^{\text {th }}$ lumbar and $1^{\text {st }}$ sacral. A craniotomy was made to expose the primary motor cortex (M1) (usually the right M1) of the hindlimb muscles located between 0 to $-1 \mathrm{~mm}$ from the Bregma and 0 to $1 \mathrm{~mm}$ from midline [28]. The dura was left intact. The exposed motor cortical area was explored with a stimulating electrode to locate the motor point from which the strongest contraction of the contralateral gastrocnemius muscle was obtained using the weakest stimuli. In experiments aimed to test the effect of dCMS on nonstimulated motor pathway, two craniotomies were made over the right and left hind limb areas of M1.

Both hind and fore limbs and the proximal end of the tail were rigidly fixed to the base. Both knees were also fixed into the base to prevent transmitting any movement from stimulated muscles to the body and vice versa. Muscles were attached to force displacement transducers (FT10, Grass Technologies, RI, and USA.) and the muscle length was adjusted to obtain the strongest twitch force (optimal length). The head was fixed in a custom made clamping system. The whole setup was placed on an anti-vibration table (WPI, Sarasota, FL, USA). Animals were kept warm during the experiment with radiant heat.

A stainless steel stimulating electrode $(500 \mu \mathrm{m}$ shaft diameter; $100 \mu \mathrm{m}$ tip) (FHC, ME, USA) was set on the exposed motor cortex. Paired stainless steel stimulating electrode ( $15 \mathrm{~mm}$ spacing; $550 \mu \mathrm{m}$ diameter) was placed on the belly of the gastrocnemius muscle, see Figure 1 (the same electrode was alternated between left and right muscles according to experimental procedure). Electrodes were then connected to stimulator outputs (PowerLab, ADInstruments, Inc, CO, USA). Extracellular recordings were made with pure iridium microelectrodes (0.180 shaft diameter; 1-2 $\mu \mathrm{m}$ tip; $5.0 \mathrm{M} \Omega$ ) (WPI, Sarasota, FL, USA). Two microelectrodes were inserted through two small openings that were carefully made into the spinal dura matter on left and right sides of the spinal cord. The insertion was made at approximately the same segmental level of the spinal cord. Reference electrodes were placed in the tissue slightly rostral to the recording sites. The ground electrodes were connected to the flap of skin near the abdomen. Motorized micromanipulators (Piezo-translator, WPI, Sarasota, FL, USA) were used to advance the microelectrodes into the ventral horns. The record of extracellular activity was passed through a standard head stage, amplified, (Neuro Amp EX, ADInstruments, Inc, CO, USA) filtered (bandpass, $100 \mathrm{~Hz}$ to $5 \mathrm{KHz}$ ), digitized at $4 \mathrm{KHz}$, and stored in the computer for further processing. A power lab data acquisition system and LabChart 7 software (ADInstruments, Inc, CO, USA) were used to acquire and analyze the data.

Once a single motoneuron was isolated at the left and right side of the spinal cord, few antidromic pulses (range, -9 to $-10 \mathrm{~V}$ ) were applied to the homonymous gastrocnemius muscle. As described by Porter [29], the presence of antidromically-evoked response with a short latency $(3.45 \mathrm{~ms})$ indicated that the recording electrode was placed in the vicinity of the neuron innervating stimulated muscle. These recordings were also used to calculate the latency of ipsilateral and contralateral spinal responses to muscle stimulation. A cortical pre-test stimulation of 10 pulses (anodal monopolar) at maximal stimulus strength (usually +8 to $+10 \mathrm{~V}$ ) was applied to the primary motor cortex (M1). Maximal stimulus strength was defined as the strength of stimulation when no further increase in muscle contraction was observed. This was also used to calculate the maximal threshold of M1 stimulation.

Next, dCMS was applied through two electrodes as shown in Figure 1. The negative output was connected to an electrode situated on the gastrocnemius muscle and the positive electrode was at M1 (Figure 1). The voltage strength and polarity were computer-controlled (LabChart, ADInstruments, Inc, CO, USA). Different combinations of stimulus parameters were tried before determining the one with the best responses. The strength of dCMS stimulation was adjusted so that contraction of the ipsilateral muscle (to M1) was at maximal strength which was reached just before the appearance of tail contraction (visually observed). This level of response was achieved by simultaneously applying a negative output (range, -2.8 to $-1.8 \mathrm{~V}$ ) to the 


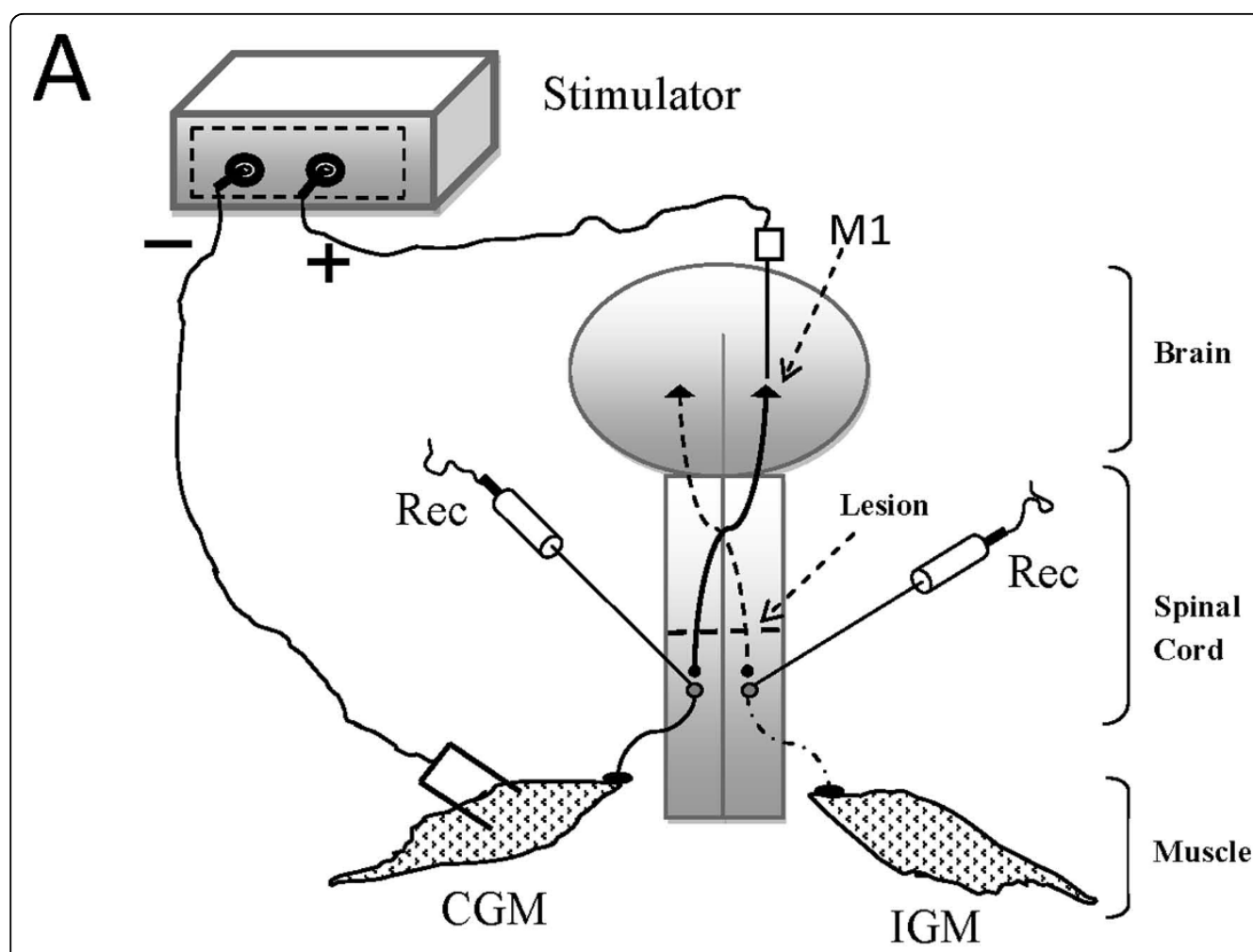

B

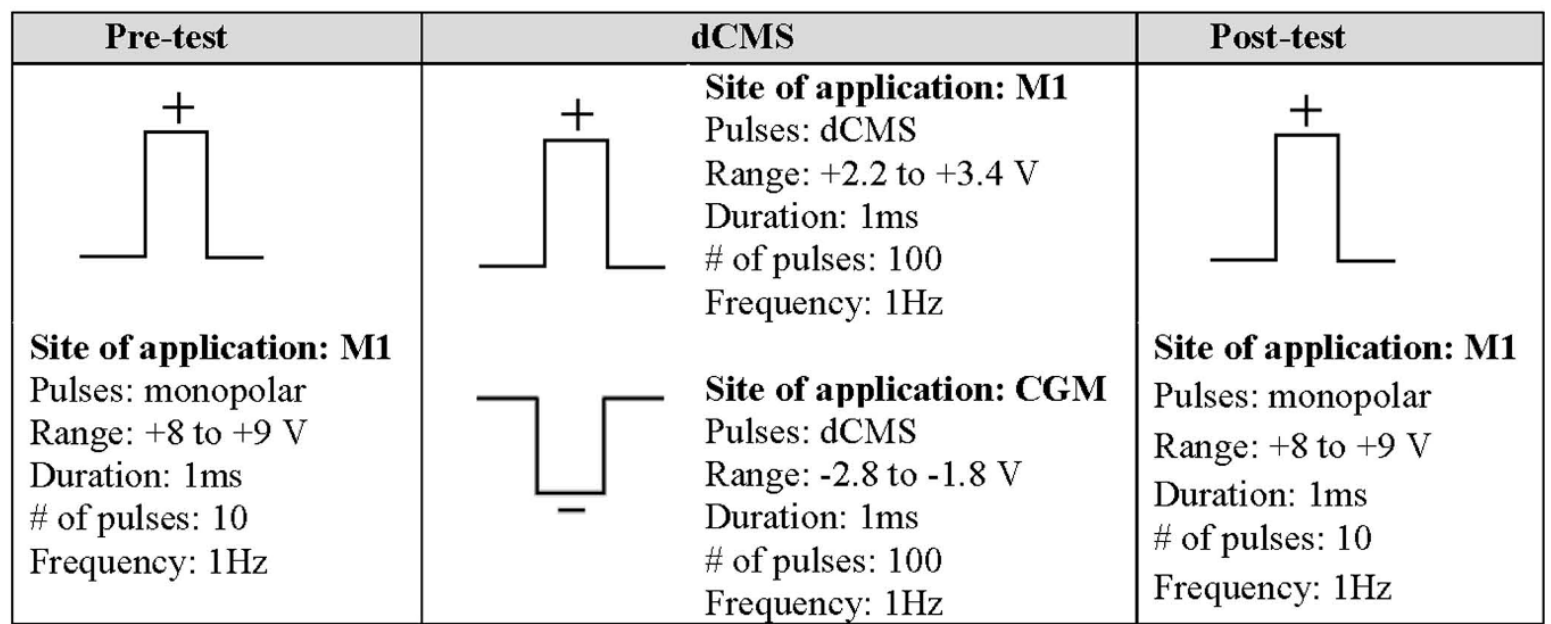

Figure 1 Experimental setup and procedure. A: The diagram illustrating the experimental set-up for dipolar cortico-muscular stimulation (dCMS). The positive and negative voltage outputs were connected to electrodes situated on the primary motor cortex (M1), and on the contralateral gastrocnemius muscle, respectively. Both gastrocnemii muscles were attached to force transducers (not shown). Recording from single motoneuron (Rec) was performed simultaneously on each side of the spinal cord below the lesion, as shown. IGM - ipsilateral gastrocnemius muscle, CGM - contralateral gastrocnemius muscle. B: The experimental procedure consisted of three phases designed to stimulate the preparation and to evaluate its reactions to dCMS. The force of muscle contraction and cortically-elicited spinal responses were evaluated before and after the application of dCMS in Pre-test and Post-test phases by application of ten monopolar pulses. The type of stimulation and location of the stimulation and recording electrodes was the same in these two phases. During dCMS phase the preparation was stimulated by application of the positive and negative pulses to the motor cortex (M1) and contralateral gastrocnemius muscle (CGM) respectively. While the number of pulses delivered during Pre- and Post-test phases was the same (10), the number of pulses delivered during dCMS was 100. The duration (1 ms) and the frequency of stimulation $(1 \mathrm{~Hz})$ were the same in all three phases of the experiment. The shape of the stimulating current at each phase is shown. There was a continuous recording of ipsilateral and contralateral muscle twitches and evoked and spontaneous spinal activity during the entire experiment. 
muscle and positive output (range, +2.2 to $+3.2 \mathrm{~V}$ ) to M1. At this maximal strength, dCMS was delivered (100 pulses, $1 \mathrm{~ms}$ pulse duration, $1 \mathrm{~Hz}$ frequency), 15 to 20 seconds after the stimulating paradigm was ended, a post-test (with identical parameters as pre-test) stimuli were delivered to M1. See Figure 1B for experimental design. Thereafter, spontaneous activity was followed for $5 \mathrm{~min}$, then the experiment was ended and animals were injected with a lethal overdose of anesthesia. In a subgroup of animals, the maximal threshold of M1 was re-tested. In addition, in this subgroup, in order to determine the duration of dCMS effect, the magnitude of cortically-elicited muscle twitches and spinal responses were retested every $20 \mathrm{~min}$ for $60 \mathrm{~min}$ after dCMS.

\section{White matter staining}

At the end of each experiment, animals were injected with a lethal dose of Ketamine. Two parts of the spinal column (including vertebrae and spinal cord) were dissected, one part $(1.5 \mathrm{~cm})$ included the lesion epicenter and another part $(\sim 0.5 \mathrm{~cm})$ included the recording area (to confirm the electrodes location). Tissues were kept overnight $\left(4^{\circ} \mathrm{C}\right)$ in $4 \%$ paraformaldehyde in $0.1 \mathrm{~m}$ PBS and cryoprotected in $20 \%$ sucrose in PBS at $4{ }^{\circ} \mathrm{C}$ for 24 $h$. The spinal column was freeze mounted and cut into $30 \mu \mathrm{m}$ sections and placed on poly-L-lysine-coated glass slides. The spinal column part including the lesion epicenter was sequentially sectioned. Slides were numbered to identify their locations relative to the lesion epicenter.

4 slides from each SCI animal $(n=6)$ containing the lesion epicenter and 2 slides containing no signs of damaged spinal cord tissue from above and below the lesion were taken for luxol fast blue (Sigma) staining. The lesion epicenter was identified as the section containing the least amount of Luxol fast blue. Sections from control animals $(n=3)$ at spinal cord T13 level were stained with luxol fast blue. Sections from the recording area were stained with cresyl violet.

The amount of spared white matter was measured using Adobe Photoshop CS4 (Adobe Systems, San Jose, CA). To assess the extent of the spinal cord damage we compared the spared white matter at the lesion epicenter with white matter at spinal cord level T13 in control animals.

\section{Data analysis}

To evaluate the latencies, we recorded the time from the start of the stimulus artifact to the onset of the first deflection of spinal response. Measurements were made with a cursor and a time meter on LabChart software. The amplitude of spinal responses was measured as peak-to-peak. Analysis of muscle contractions were performed with peak analysis software (ADInstruments, Inc,
CO, USA), as the height of twitch force measured relative to the baseline. Spike Histogram software was used to discriminate and analyze extracellular motoneuronal activity. All data are reported as group means \pm standard deviation (SD). Paired student's $t$-test was performed for before-after comparison or two sample student's $t$-test to compare two groups; statistical significance at the 95\% confidence level $(\mathrm{p}<0.05)$. To compare responses from both sides of spinal cords recorded from control animals and from animals with SCI, we performed one way ANOVA followed with Solm-Sidak post hoc analysis. Statistical analyses were performed using SigmaPlot (SPSS, Chicago, IL), Excel (Microsoft, Redwood, CA), and LabChart software (ADInstruments, Inc, CO, USA).

\section{Results}

\section{Behavioral assessment}

A contusion lesion of the spinal cord resulted in the appearance of signs of spasticity syndrome such as crossing of both limbs and fanning of the paws (compare 2A and $2 \mathrm{C}$ ). These postural changes were quantified using the abnormal pattern scale (APS). APS showed substantial increase for both on $\left(\mathrm{APS}_{\text {on }} 9.8 \pm 0.70\right)$ and off (APSoff $9.8 \pm 0.70)$ ground conditions. These postural abnormalities were also accompanied by reduction in Basso Mouse Scale (BMS) scores from 9 in control mouse to $1.2 \pm 0.47$ and $1.0 \pm 0.63$ for right and left hindlimb in SCI mouse $(\mathrm{n}=15)$, respectively. In addition, the number of errors on a horizontal ladder test was close to maximum (20) for left $(19.5 \pm 0.50)$ and right $(18.83 \pm 1.16)$ hindlimb. Collectively, these results indicate that spinal cord injury procedure used in the current study was reliable in inducing behavioral signs of the injury. This strengthens the interpretation of our data.

\section{Anatomical assessment}

Figure $2 \mathrm{~B}$ and 2D show photographs of cross-sectional slices from the thoracic spinal cord region and the lesion epicenter taken from control and SCI animals, respectively. The lesion size was proximally equal in all injured animals tested histologically $(n=6)$. A rim of white matter was spared on the lateral and ventral side of the spinal cord. The area of spared white matter at the lesion epicenter $\left(0.06 \pm 0.03 \mathrm{~mm}^{2}\right)$ was significantly reduced 16 weeks after SCI compared to the area of white matter at the same spinal level $\left(0.15 \pm 0.06 \mathrm{~mm}^{2}\right)$ in control animals $(n=3)(p=0.04, t$-test $)$, Figure 2E. On average, the total cross-sectional area (white and gray matters) of the lesion epicenter was $75 \pm 14 \%$ of the total cross-sectional area of the same spinal level in control animals.

\section{Spinal motor neuron identification}

Spinal motoneurons innervating the gastrocnemius muscle were at first identified by their large spontaneous 


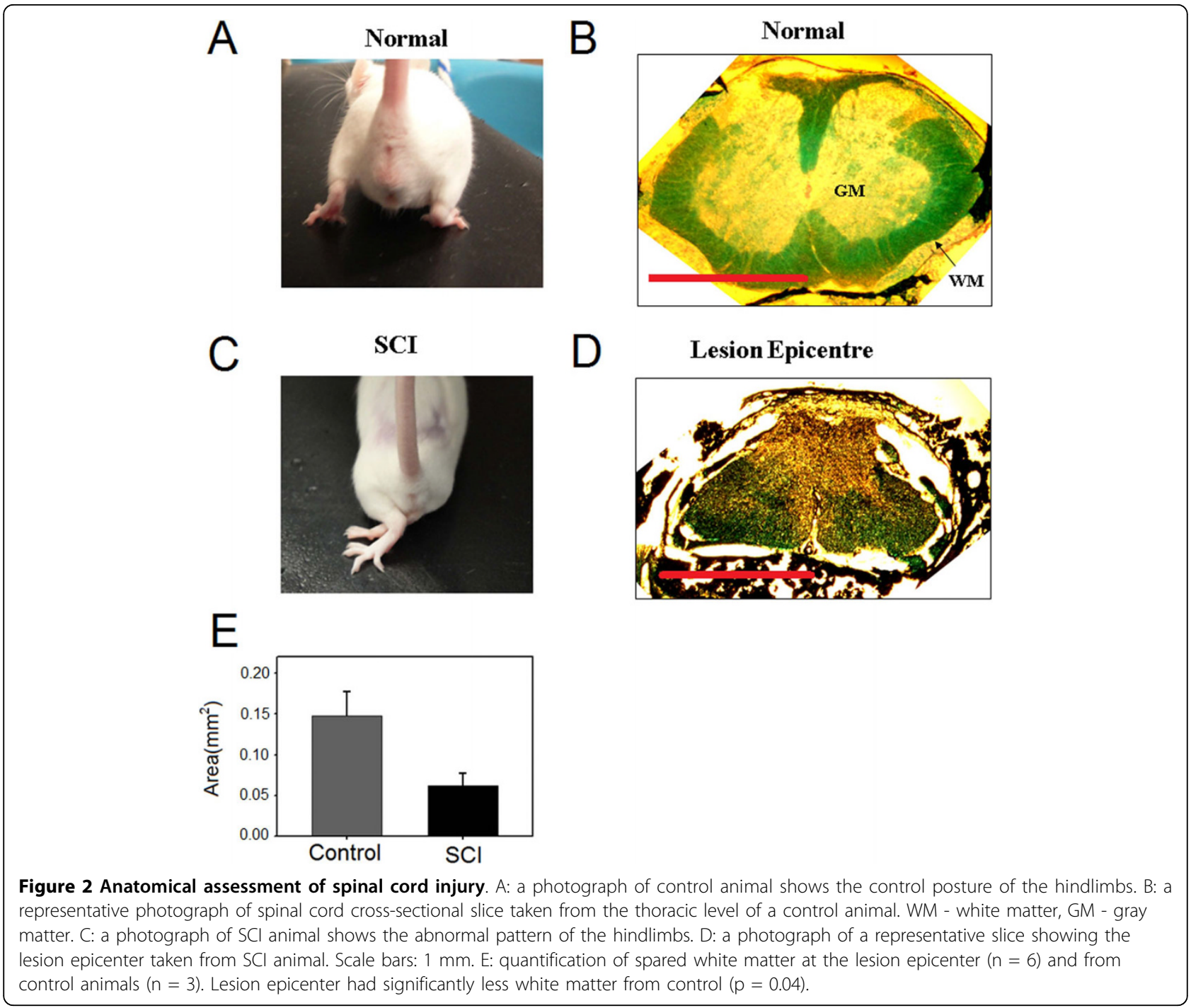

spikes. The motoneuronal spike was also accompanied by a distinctive and crisp sound recorded with a loud speaker. Second criterion used to identify spinal motoneurons was their response to the stimulation of the gastrocnemius muscle. Stimulating the gastrocnemius muscle produced a short latency antidromically-generated response that was recorded from motoneurons in the ipsilateral spinal cord. Simultaneously, the microelectrode on the contralateral side of the spinal cord recorded a response that had relatively longer latency than the one picked up from the ipsilateral side. In Figure $3 \mathrm{~A}$, three representative conditions were seen during the identification of motoneurons. The left and middle panel show simultaneous motoneuronal responses to stimulated gastrocnemius muscle. The far left panel shows the response of the motoneuron in the ipsilateral side. The middle panel shows the response of the motoneuron in the contralateral side. The far right panel shows a situation when the motoneuron was not responding to the antidromic stimulation of the homonymous gastrocnemius muscle. This confirmed that the unit was not innervating the stimulated gastrocnemius muscle. Third, as depicted in Figure 3B the muscle twitches (lower panel) were correlated with motoneuron activity (upper panel). This association between spontaneous spikes and muscle twitches was used to confirm the connection. In Figure 3B, the enlarged illustration (right) shows typical spike generated by motoneuron. Finally, we histologically confirmed that recording electrodes were localized in the ventral horn of the spinal cord.

\section{Latencies}

Stimulating the gastrocnemius muscle resulted in short and long latency spinal responses recorded by microelectrodes placed in the ipsilateral and contralateral ventral horns of the spinal cord, respectively. Figure 4A 

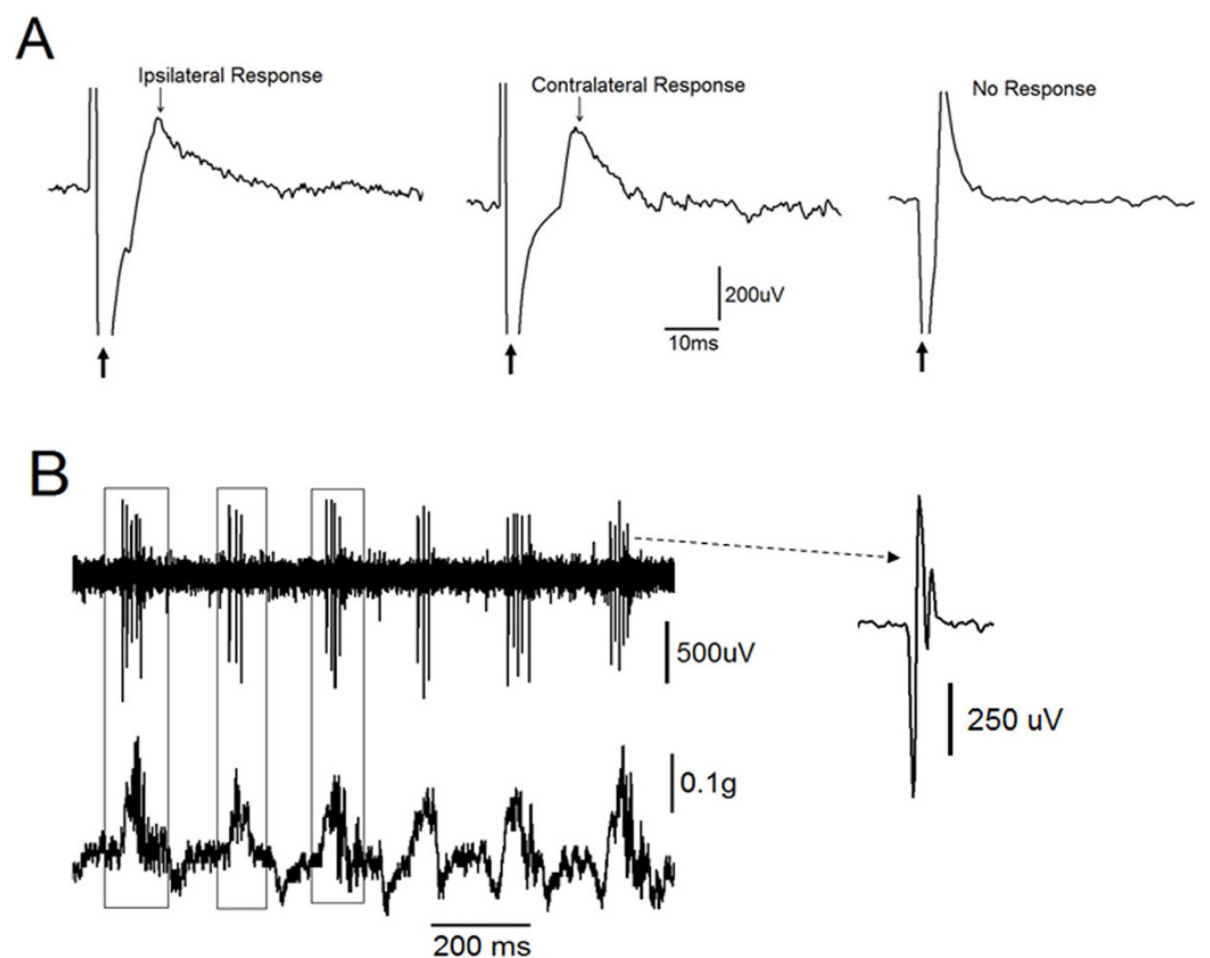

Figure 3 Identification of spinal motor neurons. A: responses to the gastrocnemius muscle stimulation. The far left and middle panels show the simultaneous responses of spinal motoneurons located ipsilateral and contralateral to the stimulated gastrocnemius muscle, respectively. The right panel shows recordings from the neuron did not respond to muscle stimulation. B: motoneurons were further identified when their spontaneous activity (upper panel) was time locked with spontaneous contractions at the ipsilateral muscle (lower panel).

shows superimposed traces of 6 antidromically-evoked responses. While the average latency of antidromicallyevoked responses was $3.45 \pm 1.54 \mathrm{~ms}$, the average latency of the contralateral responses (not shown) was longer $(5.94 \pm 1.24 \mathrm{~ms})$ indicating a transynaptic pathway. The difference between ipsilateral and contralateral spinal responses was statistically significant $(\mathrm{n}=15, \mathrm{p}<$ 0.001 , t-test). Stimulating M1 resulted in ipsilateral and contralateral spinal motoneuronal responses. Figure 4B shows six superimposed contralateral responses. The ipsilateral response is not shown in Figure 4. The average latency of ipsilateral and contralateral responses was $16.09 \pm 1.02 \mathrm{~ms}$ and $22.98 \pm 1.96 \mathrm{~ms}$, respectively. The difference in latency between ipsilateral and contralateral responses $(6.9 \mathrm{~ms})$ was statistically significant $(\mathrm{n}=$ $15, \mathrm{p}<0.001, \mathrm{t}$-test). The application of dCMS resulted in successive spinal motoneuronal responses picked up from the contralateral (to M1) electrode. Figure 4C shows six superimposed recorded traces. In this illustration (Figure 4C), three distinctive responses are seen, one with short latency $(3.45 \pm 1.54 \mathrm{~ms})$, the second with longer latency $(6.02 \pm 1.72 \mathrm{~ms})$, and a third with much longer latency $(19.21 \pm 2.28 \mathrm{~ms})(\mathrm{n}=15)$. The latency of the ipsilateral (to M1) spinal motoneuronal responses (not shown) was $6.02 \pm 2.8 \mathrm{~ms}$. Figure $4 \mathrm{D}$ summaries the average latencies collected during muscle, M1, and dCMS paradigms.

\section{Changes in cortically-elicited muscle contraction and spinal responses during dipolar cortico-muscular stimulation (dCMS)}

The application of dCMS gradually increased the twitch peak force recorded from the gastrocnemii muscles and neuronal activity recorded from the spinal cord. Since the magnitude of these enhancements were similar in control and injured animals, only data obtained from SCI animals $(n=9)$ are presented. The increase in the force of the contralateral cortically-elicited muscle contraction is shown in Figure 5 A\&5B. While Figure 5A depicts representative recordings, the averaged results obtained from all $9 \mathrm{SCI}$ animals are shown in Figure 5B. The increase from an initial twitch peak force of $4.8 \pm$ $1.12 \mathrm{~g}$ to a final twitch peak force of $6.1 \pm 0.71 \mathrm{~g}$ was statistically significant (percent change $=25.0 \pm 3.8 \%$, $\mathrm{p}=0.001$, paired $\mathrm{t}$-test). The amplitude of ipsilateral cortically-elicited muscle contraction increased as well. Representative recordings and averaged results are shown in Figure $5 \mathrm{C} \& 5 \mathrm{D}$. The final twitch force 

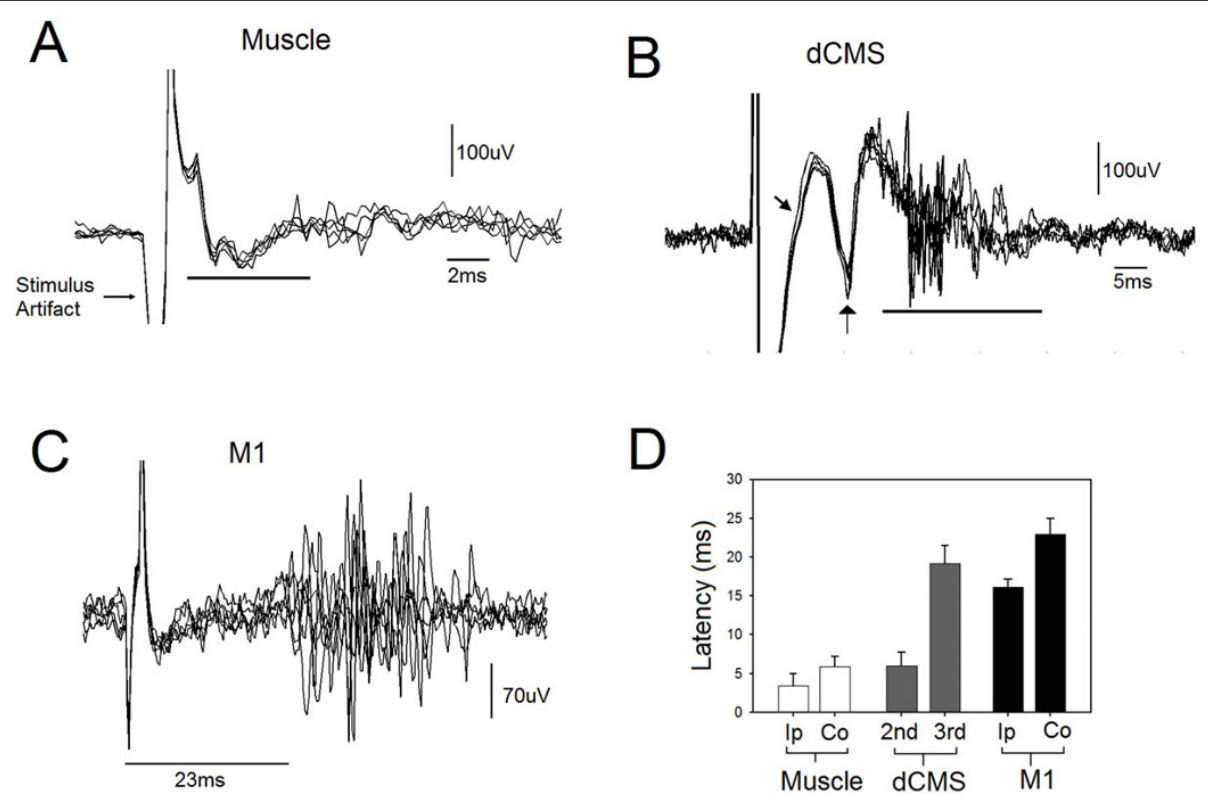

Figure 4 Spinal responses. A: six superimposed spinal responses after homonymous gastrocnemius muscle stimulation. The line marks the spinal responses. B: six superimposed spinal responses after dipolar cortico-muscular stimulation (dCMS). C: six superimposed spinal responses after motor cortex (M1) stimulation. The first and second arrows and the line mark the first, second, and third motoneuronal responses to dCMS, respectively, recorded from the contralateral spinal cord to stimulated M1. D: the average latency of spinal responses after muscle stimulation, dCMS (second and third responses), and after M1 stimulation. Ipsilateral spinal response to M1 stimulation (Ip) was significantly faster than the contralateral response $(C o)(p<0.05)$. Muscle stimulation generated significantly shorter response at ipsilateral motoneuron than the ones at the contralateral side $(p<0.05)$.
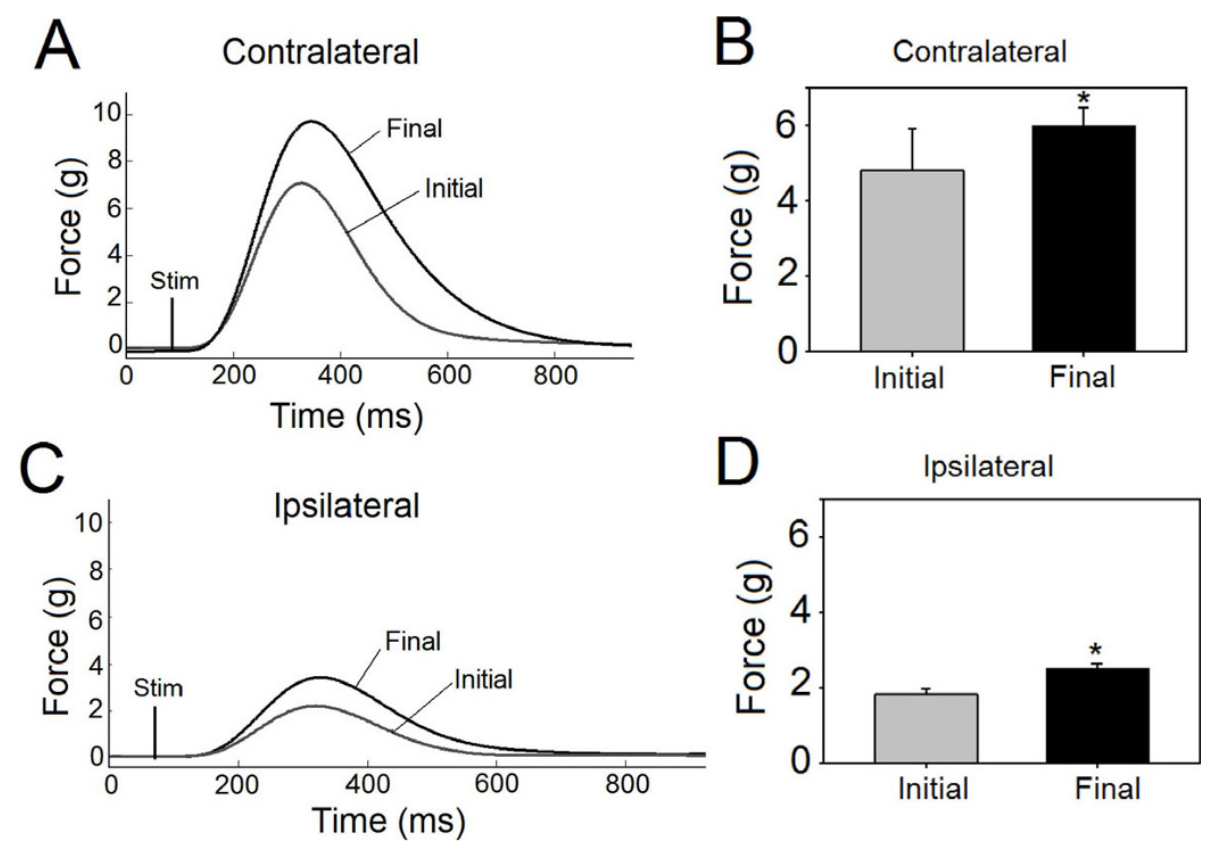

Figure 5 Muscle contraction during dipolar cortico-muscular stimulation (dCMS) in animals with $\mathbf{S C l}$. A: representative initial and final muscle twitches demonstrated greater twitch peak force at the end (final) than the beginning (initial) of dCMS on the contralateral muscle to stimulated M1. B: Bars show averages $(n=9)$ of initial and final twitch peak force of the contralateral muscle, which was significantly larger at the end of dCMS. C: representative initial and final muscle twitches of the ipsilateral muscle (to stimulated M1) during dCMS demonstrated an increase in twitch force in response to dCMS. D: bars show averages $(n=9)$ of initial and final twitch peak force of the ipsilateral muscle. ${ }^{*} p<$ 0.05. Data show means $\pm S D$. 
increased significantly from its initial value of $1.8 \pm 0.74$ g (percent change $=37.7 \pm 1.14 \% ; \mathrm{p}=0.001$, paired t-test).

Similar results were obtained by comparing the first and the last spinal motoneuronal responses of the 100 pulses of dCMS protocol. On average, the contralateral (to stimulated M1) spinal motoneuronal responses showed significant increase (percent change $=49.75 \pm$ $16.9 \%, \mathrm{p}=0.013$, one sample t-test), as did the ipsilateral (to stimulated M1) spinal motoneuronal responses (percent change $=48.10 \pm 19.8 \%, \mathrm{p}=0.04$, one sample t-test). These findings suggest that physiological processes that mediate stronger connections of the corticomotoneuronal pathway were initiated during dCMS application.

\section{The influence of dCMS application on cortically-elicited muscle twitches and neuronal activity in $\mathrm{SCl}$ animals}

We examined cortically-elicited muscle twitches (measured as peak twitch force) before and after dCMS in SCI animals. In all animals used in these experiments, twitch force was remarkably increased after dCMS. An example of twitches of the contralateral (to stimulated M1) (Figure 6A) and ipsilateral (to stimulated M1) (Figure $6 \mathrm{C}$ ) gastrocnemius muscles before (upper panels) and after (lower panel) dCMS are shown. We also examined cortically-elicited spinal responses (measured as peak - to - peak), which was also substantially increased. Examples of contralateral (Figure 6B) and ipsilateral (Figure 6D) spinal responses are shown. In Figure 6E, the twitch peak force of the contralateral muscle showed significant increase $(\mathrm{n}=9 ; \mathrm{p}<0.001)$ (average before $=0.50 \pm 0.28 \mathrm{~g} v s$. average after $=2.01$ $\pm 0.80 \mathrm{~g}$ ) (percent change $=456.1 \pm 117.5 \%$ ) after $\mathrm{dCMS}$, as did the twitch peak force of the ipsilateral (to stimulated M1) muscle (average before $=0.21 \pm 0.12 v s$. average after $=1.38 \pm 0.77, \mathrm{p}<0.001$, paired $\mathrm{t}$-test $)$ (percent change $=457 \pm 122.7 \%$ ). In Figure 6F, spinal motoneuronal responses $(n=9)$ contralateral (to stimulated M1) showed significant increase after dCMS (average before $=347.67 \pm 294.68 \mu \mathrm{V} v s$. average after $=$ $748.90 \pm 380.59 \mu \mathrm{V}, \mathrm{p}=0.027$, paired t-test) (increased by $313 \pm 197 \%$ ), as did ipsilateral (to stimulated M1) spinal motoneuronal responses (average before $=307.13$ $\pm 267.27 \mu \mathrm{V} v s$. average after $=630.52 \pm 389.57 \mu \mathrm{V}, \mathrm{p}=$ 0.001 , paired t-test) (increased by $292 \pm 150 \%$ ). Data are shown as means \pm SD. These results show that dCMS greatly potentiates the corticomotoneuronal pathway in injured animals.

The maximal cortical threshold defined as the lowest electrical stimulus eliciting the strongest muscle twitch peak force was reduced from $9.4 \pm 0.89 \mathrm{~V}$ to $=5.7 \pm$ $0.95 \mathrm{~V}$ after dCMS application $(\mathrm{n}=4, \mathrm{p}<0.001$, $\mathrm{t}$-test). The cortically-elicited muscle twitch force and the magnitude of spinal motoneuronal responses, evaluated 60 min after dCMS in 5 SCI animals, were still significantly elevated on both sides $(\mathrm{p}<0.001)$.

\section{Effects of dCMS on the non-stimulated corticomotoneuronal pathway in animals with $\mathrm{SCl}$}

The test stimulation of the other M1, contralateral to M1 where dCMS had been applied, revealed an increase of the contraction force recorded from contralateral and ipsilateral gastrocnemii muscles. The increase in contralateral (percent change $=182.8 \pm 87.18 \%$ ), and ipsilateral muscles (percent change $=174.8 \pm 138.91 \%$ ) was statistically significant ( $\mathrm{n}=6, \mathrm{p}<0.05$, t-test).

Contralateral spinal motoneuronal response was increased significantly ( $\mathrm{p}=0.006, t$-test) (average percent change $=373.8 \pm 304.99 \%$ ), as did ipsilateral (average percent change $=289.2 \pm 289.62 \%, \mathrm{p}=0.025$, $t$-test). These results indicate that even though dCMS was unilaterally applied, it affected the corticomotoneuronal pathway bilaterally.

\section{The influence of dCMS application on cortically-elicited} muscle twitches and neuronal activity in control animals The application of dCMS across the corticomotoneuronal pathway in control animals $(n=6)$ resulted in an increase in the cortically-elicited muscle contraction force produced by both gastrocnemii muscles. The twitch peak force of the contralateral muscle increased from $1.62 \pm 1.0 \mathrm{~g}$ before to $5.12 \pm 1.67$ after dCMS application (percent change $=250.75 \pm 129.35 \%, \mathrm{p}=$ 0.001 , paired t-test, Figure 7A). The twitch peak force of the muscle on the ipsilateral side increased as well, although the increase was less pronounced (from $0.16 \pm$ $0.05 \mathrm{~g}$ to $0.39 \pm 0.08 \mathrm{~g}$ ), before and after $\mathrm{dCMS}$, respectively (percent change $=166.38 \pm 96.56 \%, p=0.001$, paired t-test, Figure 7A)

The amplitude of evoked responses recorded from spinal motoneurons was also enhanced by dCMS application. As depicted in Figure 7B, the average amplitude of these spikes recorded at the contralateral side increased from $127.83 \pm 46.58 \mu \mathrm{V}$ to $391.17 \pm 168.59$ $\mu \mathrm{V}$ (percent change $=168.83 \pm 152.00 \%, \mathrm{p}=0.009$, paired t-test). The increase at the ipsilateral side was even greater (percent change $=369.00 \pm 474.00 \%, 77.50$ $\pm 24.73 \mu \mathrm{V}$ before versus $267.00 \pm 86.12 \mu \mathrm{V}$ after $\mathrm{dCMS}, \mathrm{p}=0.007$, paired $\mathrm{t}$-test).

\section{Comparison between control and $\mathrm{SCl}$ animals}

The cortically-elicited muscle twitches of contralateral muscle, recorded from control animals were stronger than twitches observed in SCI animals regardless of whether they were recorded before $(\mathrm{p}=0.009$, $\mathrm{t}$-test), or after ( $\mathrm{p}=0.001, \mathrm{t}$-test) the dCMS procedure. The response of ipsilateral muscles, however, was more 


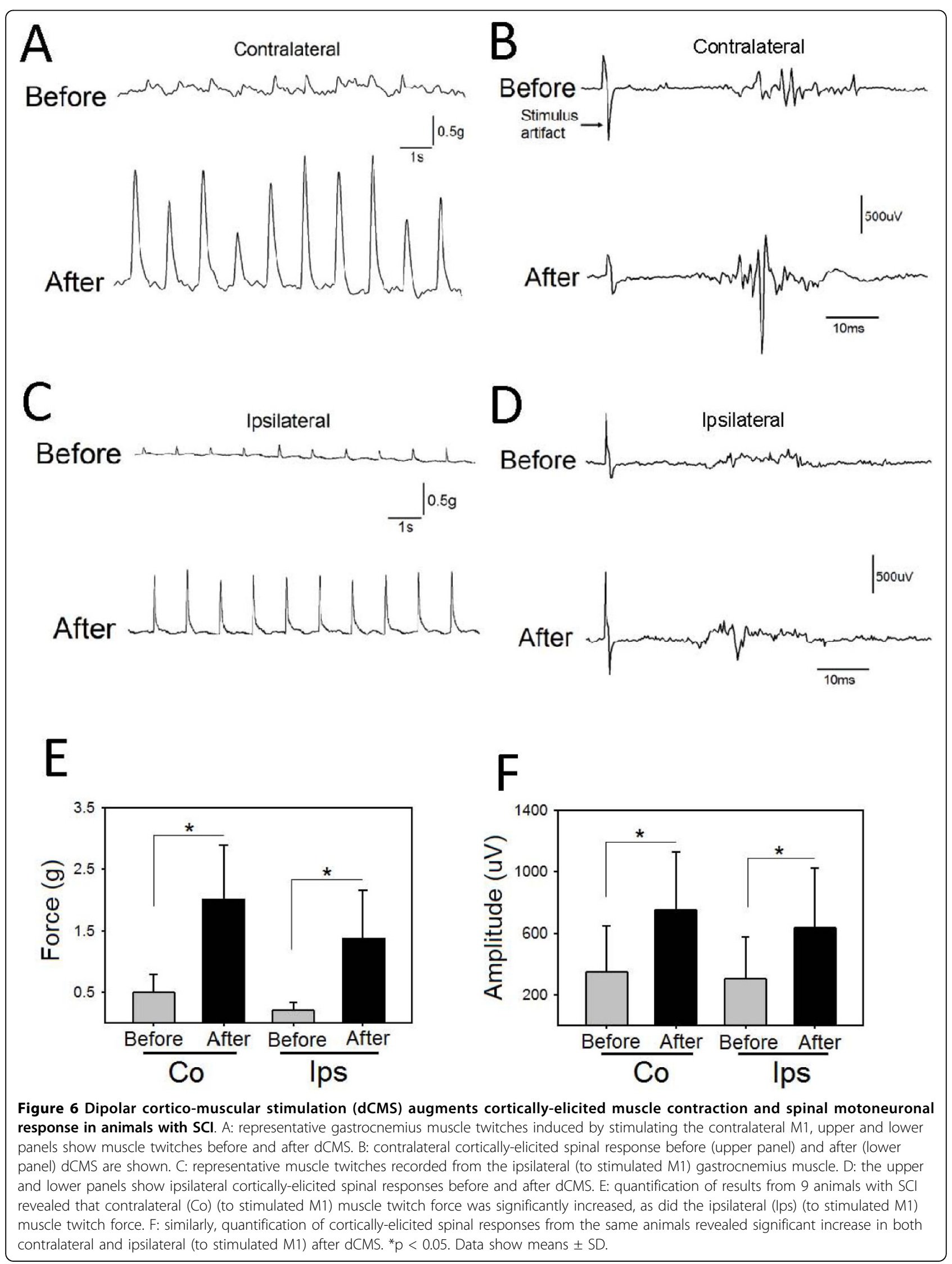



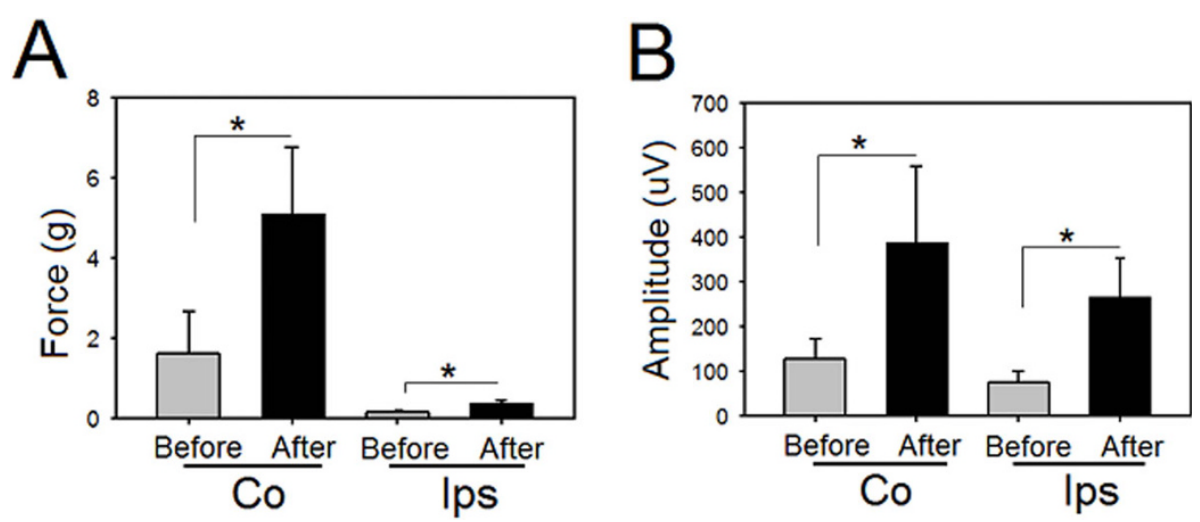

Figure 7 Cortically-elicited muscle contraction and spinal responses after dipolar cortico-muscular stimulation (dCMS) in control mice. A: quantification of results from 6 control animals revealed significant increase in contralateral (CO) and ipsilateral (Ips) (to stimulated M1) muscle twitch force after dCMS. B: contralateral (to stimulated M1) cortically-elicited spinal responses were significantly increased after dCMS, as did ipsilateral responses. ${ }^{*} p<0.05$. Data show means \pm SD.

complex. Before dCMS, SCI animals showed higher ipsilateral twitch peak force than control animals, although the difference was not statistically significant $(\mathrm{p}=0.39$, t-test). This difference became statistically significant after dCMS intervention ( $\mathrm{p}=0.01$, $\mathrm{t}$-test).

Similarly, before dCMS, the cortically-elicited responses recorded from spinal motoneurons were higher in SCI animals at ipsilateral and contralateral sides, although the difference did not reach statistical significance $(\mathrm{p}=0.13, \mathrm{t}$-test $)$. However, following dCMS, this difference was increased and became statistically significant $(\mathrm{p}=0.009, \mathrm{t}$-test $)$.

Next we have calculated a relative measure of muscle performance, which we called "fidelity index" (FI). FI is the ratio of cortically-elicited spinal motoneuronal response to the corresponding muscle twitch peak force (spinal response/muscle twitch ratio). Lower fidelity index value indicates better association between spinal responses and their corresponding muscle twitches. In other words, it means better ability of a spinal response to induce muscle contraction. Therefore, changes in this index may indicate changes in relation between spinal and peripheral excitability.

After dCMS, SCI animals showed overall significant group reduction in FI $(\mathrm{F}=3.3, \mathrm{p}<0.033$, ANOVA $)$ (Figure 8). Solm-Sidak post hoc test showed reduction in contralateral FI (average before $=368.35 \pm 342.51$ vs. average after $=246.15 \pm 112.24$ ), however, the difference was not statistically significant $(p=0.46)$. The ipsilateral FI was significantly reduced after dCMS (average before $=704.59 \pm 625.7 v s$. average after $=247.95 \pm 156.27)(\mathrm{p}$ $=0.011$ ). The effect of dCMS treatment was the opposite in control animals which demonstrated overall group increase in $\mathrm{FI}$ after this procedure $(\mathrm{F}=31.51, \mathrm{p}<$ 0.001 , ANOVA). FI was significantly increased after
dCMS (Solm-Sidak post hoc, $\mathrm{p}<0.001$ ) in the ipsilateral side (average before $=328.53 \pm 104.83$ vs. average after $526.83 \pm 169.38$ ). There was also a trend reflecting an increase in the contralateral side (average before $=48.59$ $\pm 17.71 \mathrm{vs}$. average after $=56.15 \pm 24.19$ ), but was not statistically significant (Solm-Sidak post hoc, $\mathrm{p}=0.89$ ).

Comparing FI from control animals with FI from SCI animals showed a statistically significant lower index in

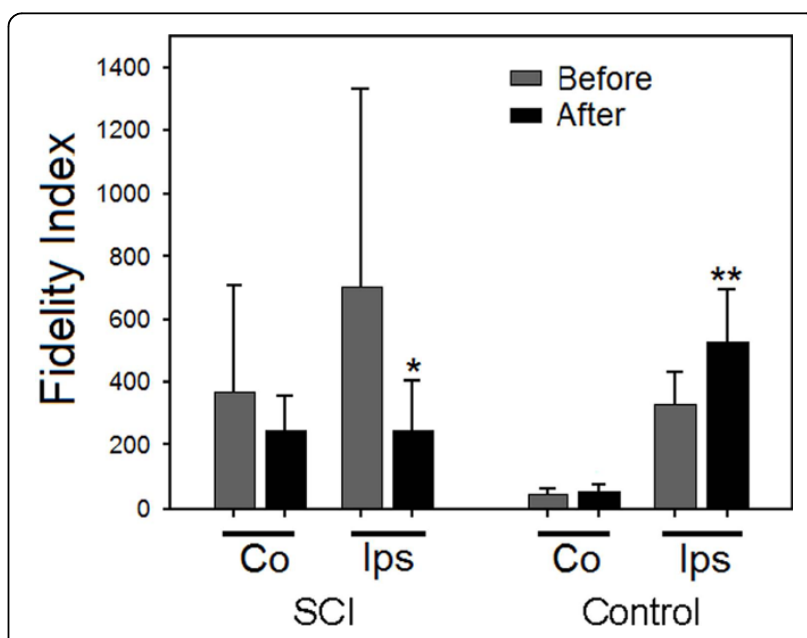

Figure 8 Fidelity index analysis. Fidelity index (spinal response/ muscle twitch force) was quantified from 6 control and 9 with $\mathrm{SCl}$ animals. In animals with $\mathrm{SCl}$, Contralateral (CO) to stimulated M1 fidelity index shows reduction after dCMS but was not statistically significant; however, ipsilateral (Ips) fidelity index was significantly reduced after dCMS. In control animals, after dCMS, Fidelity index was increased contralateral to stimulated M1 but was not statistically significant, however, the ipsilateral Fidelity index was significantly higher after dCMS. Note that the lower the fidelity indexes the better the correlation between muscle contraction and spinal response. ${ }^{*} p<0.05$, lower from before $d C M S$; ${ }^{* *} p<0.05$, higher from before dCMS. Data show means \pm SD. 
the contralateral side of control animals $(\mathrm{p}<0.001$, ANOVA, Solm-Sidak post hoc) both before and after dCMS. These results support the findings that peripheral nerves are in-excitable or of higher threshold in subjects with SCI [13].

dCMS increased spinal motoneurons spontaneous activity Comparing the firing rate of spontaneous activity before and after dCMS intervention demonstrated significant increase in both control and SCI animals. In Figure 9 A\&9B, a representative spontaneous activity recording from an SCI animal is shown. In SCI animals, spontaneous activity was significantly increased in the contralateral side of the spinal cord (average before $=17.31 \pm$ $13.10 \mathrm{spikes} / \mathrm{s} v \mathrm{~s}$. average after $=32.13 \pm 14.73 \mathrm{spikes} / \mathrm{s}$; $\mathrm{p}=0.001)(121.71 \pm 147.35 \%)$, as it did in the ipsilateral side (average before $=18.85 \pm 13.64$ spikes $/ \mathrm{s} v$ s. average after $=26.93 \pm 17.25 ; \mathrm{p}=0.008)($ percent change $=$ $54.10 \pm 32.29 \%)$. In control animals, spontaneous activity was significantly increased in the contralateral (to

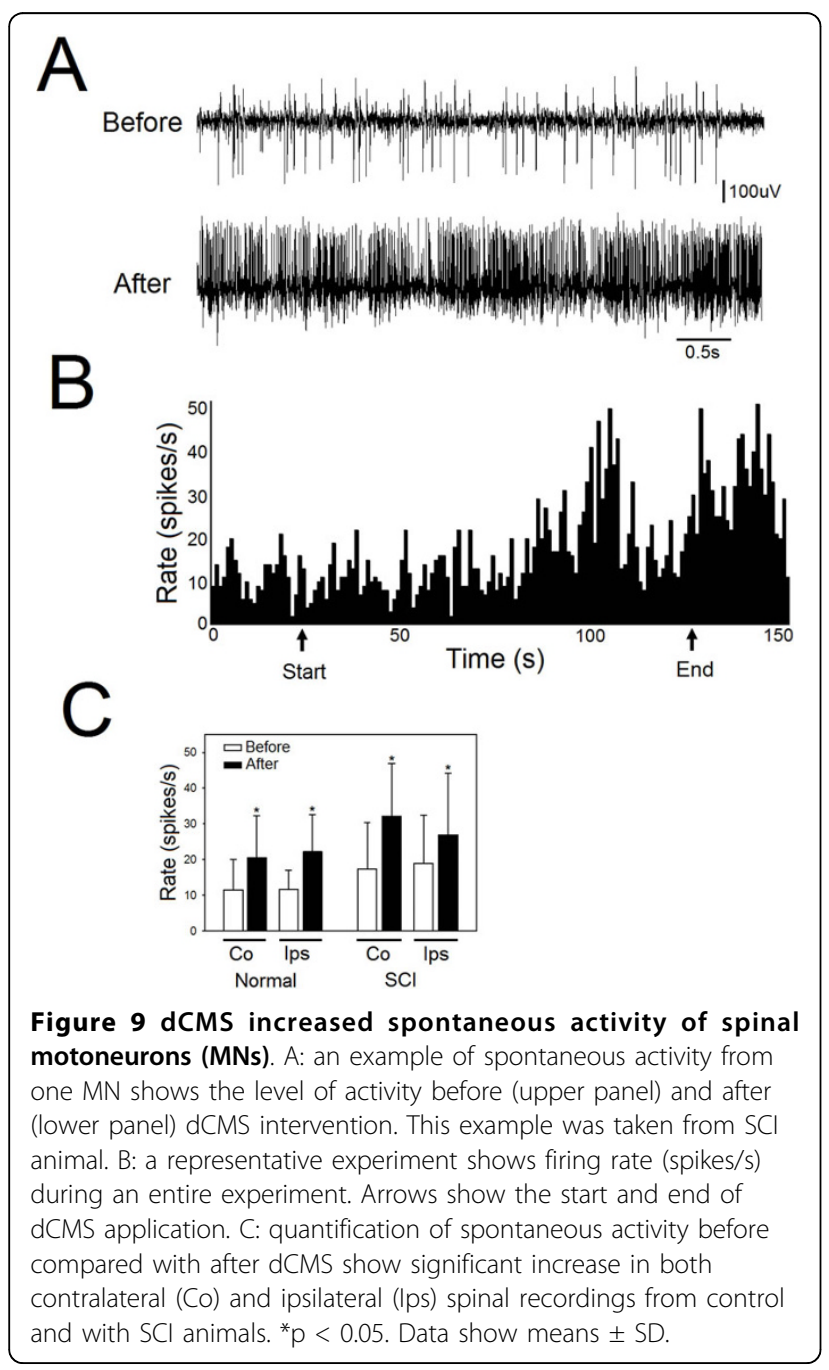

stimulated M1) side of the spinal cord (average before = $11.40 \pm 8.65$ spikes $/ \mathrm{s} v s$. average after $=20.53 \pm 11.82$ spikes/s; $\mathrm{p}=0.006)$ (percent change $=90.10 \pm 42.53 \%$ ), as it did in the ipsilateral side (average before $=11.63 \pm$ 5.34 spikes $/ \mathrm{s} v s$. average after $=22.18 \pm 10.35$ spikes $/ \mathrm{s}$; $\mathrm{p}=0.01$ ) (percent change $=99.10 \pm 1.10 \%$ ). One way ANOVA showed no significant difference between control and SCI animals in firing rate, although, SCI animals demonstrated higher firing rate.

\section{Effects of monopolar stimulation of muscle or cortex}

In order to determine that the effect was unique to dCMS, the influence of monopolar stimulation (maximal stimulation for 100 pulses, $1 \mathrm{~Hz}$ frequency) of either the muscle or the motor cortex on spinal motoneuronal response and muscle twitch peak force was examined.

As expected, muscle stimulation resulted in significant reduction in muscle twitch force $(-20.28 \pm 7.02 \%$, $\mathrm{p}<$ $0.001, \mathrm{t}$-test) ( $\mathrm{n}=5,3 \mathrm{SCI}$ and 2 control). It also resulted in a significant reduction in spinal motoneuronal responses evoked by the contralateral (to stimulated muscle) $\mathrm{M} 1$ test stimulation (average before $=747.50 \pm$ $142.72 \mu \mathrm{V}, v s$. average after $=503.14 \pm 74.78)(\mathrm{F}=$ 17.11, one way ANOVA, Solm-Sidak post hoc, p < 0.001 ), however, no significant change was seen in responses recorded in the ipsilateral (to stimulated muscle) side of the spinal cord (average before $383.33 \pm$ $140.67 \mu \mathrm{V} v s$. average after $=371.43 \pm 35.61, \mathrm{p}=0.84)$.

In a separate group of animals $(\mathrm{n}=5,3 \mathrm{SCI}$ and 2 control), we also tested the effect of the monopolar stimulation paradigm applied only at the motor cortex on contralateral muscle twitch peak force and spinal motoneuronal response. Both, the muscle twitch and motoneuron response were significantly reduced by over $50 \%$ $(-53.69 \pm 4.3 \%, \mathrm{p}=0.001, \mathrm{t}$-test $)$ and almost $15 \%(-14.59$ $\pm 9.10 \%, \mathrm{p}=0.003, \mathrm{t}$-test), respectively. These results indicate that monopolar muscle or cortical stimulation at maximal strength results in fatigue of muscle twitch force and reduction in spinal responses.

\section{Discussion}

The results show remarkable enhancement of the excitability of the corticomotoneuronal pathway induced by unilateral application of the dCMS. This enhancement was observed in control animals and in SCI animals that had severe locomotor impairment associated with signs of spastic syndrome. The effect was observed both in the ipsilateral and contralateral pathways. The maximal threshold of the ipsilateral cortex was reduced. Improvement in muscle strength was accompanied by an increase in spontaneous activity and potentiation of evoked responses of the spinal motoneurons. The spinal motoneuronal responses and muscle twitches evoked by the stimulation of the contralateral, non-treated M1 
were significantly enhanced as well. The dCMS-induced effect persisted beyond the phase of stimulation and extended through the entire period of the experiment (60 min).

Bilateral responses to cortical stimulation have been routinely observed [3,6,30-33]. They can be mediated by interhemispheric connections, ipsilateral cortico-spinal connections (5-6\% of the contralateral projections) [34], or commissural spinal neurons. As seen in Figure 6F and $7 \mathrm{~B}$, ipsilateral responses to unilateral stimulation of motor cortex evoked larger responses in SCI animals compared to controls. These results further support the idea that ipsilateral corticospinal projections are more efficient in evoking muscle contraction after SCI [3].

The mechanism of the dCMS induced increase in the excitability of the corticomotoneuronal pathway is not clear and one can only speculate as to what processes have been modulated. It is obvious that the potentiation in cortically-elicited muscle contraction during dCMS is not like the potentiation seen after neuromuscular stimulation [35]. While neuromuscular stimulation leads to a brief potentiation of muscle force followed by a steep reduction in force, dCMS leads to a gradually proceeding increase in the amplitude of cortically-elicited muscle contraction. Since the enhancement occurred at contra- and ipsilateral sides, the locus of potentiation is most likely either spinal or supraspinal. The enhancement of cortically-elicited muscle contraction was accompanied by a reduction in maximal threshold to cortical stimulation, an increase in spinal motoneuronal responses, and an increase in cortically-elicited spinal motoneuronal responses. Therefore, one can assume that improvements occurred simultaneously at several functional levels of the corticomotoneuronal pathway.

In view of the fact that the current employed in our stimulation paradigm was always positive at one end and negative at the other, our stimulation can be considered in part polarizing. The paradigm of polarizing current was used to study excitability of different parts of the nervous system [36-39]. In these studies, polarizing current produced potential membrane changes in which hyperpolarization occurs at cellular parts near the positive electrode and depolarization occurs near the negative electrode. Complying with this rule, for example, the situation of two polarizing electrodes on the spinal cord (one on the ventral side and the other on the dorsal side) produced changes in membrane and spike potentials of primary fibers from muscles [36]. In our study we suggest that the current is polarizing during the brief, steady moment of pulse duration $(1 \mathrm{~ms})$. Given the electrodes placement, in which negative at the muscle and positive at the cortex, the cell body of corticospinal neurons is expected to hyperpolarize and their nerve terminals depolarize. Moreover, spinal motoneurons expected to hyperpolarize at the cell body and dendrites, and depolarize at the neuromuscular junction. According to cell topography relative to the applied electrical field, membrane potential changes are also expected to occur at intervening interneurons. These membrane changes that occur briefly during each pulse of dCMS, seem to prime corticomotoneuronal pathway for potentiation. In addition, the stimulating pulse has two more periods: rising $(0.250 \mathrm{~ms})$ and falling $(0.250 \mathrm{~ms})$. These changing periods caused a flow of current that exited from one end and entered at the other end of the corticomotoneuronal pathway. This idea is supported by the observation of stimulus artifact picked up by electrodes in the spinal cord. The current flowed throughout the entire pathway independent from the factors confounding active excitability (see introduction). This might cause activation of the corticomotoneuronal pathway at any possible excitable site/s. This will ensure eliciting spike-timing-dependent plasticity [40] that might be one of the mechanisms that mediates the effect of the dCMS. In addition, the high frequency multiple spinal responses, evoked during dCMS, can, in principle, induce long-term potentiation [41]. Because dCMS can engage a variety of neuronal mechanisms as well as non-neuronal activity, its effect might be a combination of many changes along the corticomotoneuronal pathway.

The dCMS-induced enhancement of cortically-elicited muscle contraction has been observed in both - control and injured animals. The mechanisms responsible for this amplification in these two groups of animals may overlap, but they do not have to be identical. Although, as discussed above the potentiating effect of dCMS could be mediated by strengthening synaptic responses, the nature and source of these changes may differ substantially in the corticomotoneuronal pathway of control and injured animals. Axonal sprouting is probably the primary source of synaptic connections in the damaged spinal cord [1-3]. However, axonal sprouting does not grant the formation of functional connections. Therefore, one of the probable mechanisms that may mediate the potentiating effect of dCMS is the refining and strengthening of the weak synaptic connections that have resulted from sprouting. Moreover, dormant connections that exist throughout the sensorimotor system [6] may be activated and become functional after dCMS. Potentiating the spared normal connections could also happen after dCMS. On the other hand, in control animals, potentiating normal connections and facilitating dormant connections might be the only processes that mediate the effect of dCMS. The results show that dCMS stimulation was almost twice as effective in injured animals compared with controls. This indicates that injured spinal cord is more prone for dCMS 
stimulation and posses extra mechanisms mediating the dCMS effect.

In SCI animals, even before the application of dCMS, the spinal motoneurons were responding more aggressively to cortical stimulation than were controls. Nevertheless, very weak or no muscle contraction was seen (Figure 6). This might be due to one of two mechanisms. One would be located in the spinal cord caudal to the lesion and/or the other being, the inexcitable peripheral nerves and/or the irresponsiveness of the muscle. Caudal to the lesion, the activity of the spinal motoneuron pool was probably desynchronized as a result of reorganization. Supporting this idea are the findings by Brus-Ramer and colleagues [3]. The authors reported that chronic stimulation of corticospinal tracts resulted in preferential axonal outgrowth toward the ventral horn. This indicates that inter motoneuronal connections are dynamic processes, which may change by decentralization. Inexcitable peripheral axons were found in patients with SCI [13]. Assuming that the axons in SCI animals are in similar conditions, they could experience an action potential failure resulting in reduced muscle contraction. Muscle atrophy is always seen in animals with SCI $[9,10$, and 12] and humans [11]. This might also be one of the reasons why spinal motoneurons responses were not translated adequately into muscle contraction. We quantified the adequacy of motoneuronal responses by calculating the fidelity index, which is the ratio of spinal response to muscle twitch force. The dCMS-induced changes in the fidelity index were opposed in control and injured animals. While this index has been reduced in injured animals, indicating improvement in the effectiveness of the corticomotoneuronal pathway, it had increased in control animals suggesting lowering of the pathway effectiveness probably due to fatigue interference. Therefore, one can imply that injury to the spinal cord initiates processes which favor regeneration of the function. Apparently our procedure synchronizes and facilitates these processes, promoting recovery.

It has been demonstrated that spontaneous activity in spinal motoneurons is a significant factor in developing spinal circuits involved in locomotion [42,43]. It has also been shown that increasing or decreasing the frequency of spontaneous activity will disrupt connectivity in a developing spinal cord $[44,45]$. In the light of these studies and our data one can ask what role the changes in spinal motoneuronal spontaneous activity play in recovery after SCI, and what are the interactions between interventions, spontaneous activity and functional recovery after SCI? These questions await further investigations which could be guided by our observation that dCMS increased the tonic activity of spinal motoneurons in animals with SCI as well as in control animals.
Before the dCMS application, the spontaneous activity of motoneurons in animals with SCI was higher than that of control animals. This and the exaggerated evoked spinal responses in animals with SCI, is consistent with the behavioral measurements that show spastic syndrome-like characteristics. The exaggerated spontaneous firing rate of spinal motoneurons is also consistent with data from motor unit firing in humans and animals after SCI $[46,47]$ and with results from intracellular recordings from sacrocaudal motoneurons that show a sustained and exaggerated firing rate in animals with SCI [48]. Minutes after dCMS, motoneuronal spontaneous activity was still substantially increased. Some of these activities were rhythmic, as shown in Figure 3B, although most of the spontaneous activity was in an unmodulated pattern of firing as shown in Figure 9A. Voltage-dependent persistent inward currents (PICs) that strengthen synaptic inputs in normal behavior depend on descending brain-stem-released serotonin (5-HT) or noradrenalin [49-51]. Here the increase in the spontaneous firing rate and the appearance of modulated activity in some animals after dCMS may indicate better connections with brain-stem centers.

In conclusion, the results showed clear evidence that dCMS is an effective method that enhances the excitability of the corticomotoneuronal connections. This technique has the potential to be used in humans suffering after spinal cord injury, stroke, multiple sclerosis, and others. In practice, it can be employed to strengthen or awaken any weak or dormant pathway in the nervous system.

\section{Acknowledgements}

This research was supported by NYS/DOH grant \# CO23684 and PSCCUNY grant 60027-37-39.

\section{Competing interests}

Currently applying for a patent relating to the content of the manuscript.

Received: 24 March 2010 Accepted: 17 September 2010

Published: 17 September 2010

\section{References}

1. Murray M, Goldberger ME: Restitution of function and collateral sprouting in the cat spinal cord: the partially hemisected animal. J Comp Neurol 1974, 158(1):19-36.

2. Bareyre FM, Kerschensteiner M, Raineteau O, Mettenleiter TC, Weinmann O, Schwab ME: The injured spinal cord spontaneously forms a new intraspinal circuit in adult rats. Nat Neurosci 2004, 7:269-77.

3. Brus-Ramer M, Carmel JB, Chakrabarty S, Martin JH: Electrical stimulation of spared corticospinal axons augments connections with ipsilateral spinal motor circuits after injury. J Neurosci 2007, 27:13793-13901.

4. Miles R, Wong RK: Latent synaptic pathways revealed after tetanic stimulation in the hippocampus. Nature 1987, 329:724-6.

5. Fregni F, Boggio PS, Valle AC, Rocha RR, Duarte J, Ferreira MJ, Wagner T, Fecteau S, Rigonatti SP, Riberto M, Freedman SD, Pascual-Leone A: A shamcontrolled trial of a 5-day course of repetitive transcranial magnetic stimulation of the unaffected hemisphere in stroke patients. Stroke 2006, 37:2115-22. 
6. Brus-Ramer M, Carmel JB, Martin JH: Motor cortex bilateral motor representation depends on subcortical and interhemispheric interactions. J Neurosci 2009, 29:6196-206.

7. Ridding MC, Taylor JL: Mechanisms of motor-evoked potential facilitation following prolonged dual peripheral and central stimulation in humans. J Physiol 2001, 537:623-31.

8. Huerta PT, Volpe BT: Transcranial magnetic stimulation, synaptic plasticity and network oscillations. J Neuroeng Rehabil 2009, 2:6-7.

9. Ahmed Z, Wieraszko A: Combined effects of acrobatic exercise and magnetic stimulation on the functional recovery after spinal cord lesions. J Neurotrauma 2008, 25:1257-1269.

10. Liu M, Bose P, Walter GA, Thompson FJ, Vandenborne K: A longitudinal study of skeletal muscle following spinal cord injury and locomotor training. Spinal Cord 2008, 46:488-93.

11. Shah PK, Stevens JE, Gregory CM, Pathare NC, Jayaraman A, Bickel SC, Bowden M, Behrman AL, Walter GA, Dudley GA, Vandenborne K: Lowerextremity muscle cross-sectional area after incomplete spinal cord injury. Arch Phys Med Rehabil 2006, 87:772-778.

12. Gordon T, Mao J: Muscle atrophy and procedures for training after spinal cord injury. Phys Ther 1994, 74:50-60.

13. Lin CS, Macefield VG, Elam M, Wallin BG, Engel S, Kiernan MC: Axonal changes in spinal cord injured patients distal to the site of injury. Brain 2007, 130:985-994

14. Curt $A$, dietz V: Electrophysiological recordings in patients with spinal cord injury: significance for predicting outcome. Spinal Cord 1999, 37:157-65

15. Tillakaratne NJ, Mouria M, Ziv NB, Roy RR, V Edgerton R, Tobin AJ: Increased expression of glutamate decarboxylase (GAD (67)) in feline lumbar spinal cord after complete thoracic spinal cord transection. J Neurosci Re 2000, 60:219-230.

16. Knikou M: Neural control of locomotion and training-induced plasticity after spinal and cerebral lesions. Clin Neurophysiol 2010, 12:1655-1668.

17. Raineteau O, Schwab ME: Plasticity of motor systems after incomplete spinal cord injury. Nat Rev Neurosci 2001, 2:263-73.

18. Hebb D: organization of behavior. New York: Wiley 1949.

19. Levy WB, Steward O: Temporal contiguity requirements for long-term associative potentiation/depression in the hippocampus. Neuroscience 1983, 8:791-797.

20. Bliss TVP, Collingridge GL: A synaptic model of memory: Long-Term Potentiation in the hippocampus. Nature 1993, 361:31-39.

21. Eccles JC, Kostyuk PG, Schmidt RF: The effect of electric polarizing of the spinal cord on central afferent fibres and on their excitatory synaptic action. J Physiol 1962, 162:138-150

22. Nitsche MA, Paulus W: Excitability changes induced in the human motor cortex by weak transcranial direct current stimulation. J Physiol 2000, 527:633-639

23. Gruner JA: A Monitored contusion model of spinal cord injury in the rat. J Neurotrauma 1992, 9:123-126.

24. Engesser-Cesar C, Anderson AJ, Basso DM, Edgerton VR, Cotman CW: Voluntary wheel running improves recovery from a moderate spinal cord injury. J Neurotrauma 2005, 22:157-171.

25. Zandieh S, Hopf R, Redl H, Schlag MG: The effect of ketamine/xylazine anesthesia on sensory and motor evoked potentials in the rat. Spinal Cord 2003, 41:16-22.

26. Hicks RG, Woodforth IJ, Crawford MR, Stephen JP, Burke DJ: Some effects of isoflurane on I waves of the motor evoked potential. $\mathrm{Br} J$ Anaesth 1992, 69:130-136.

27. Sloan TB: Anesthetic effects on electrophysiologic recordings. J Clin Neurophysiol 1998, 15:217-226.

28. Franklin KBJ, Paxinos G: The Mouse Brain in Stereotaxic Coordinates. Academic Press, 32007.

29. Porter R: Early facilitation at corticomotoneuronal neuronal synapses. J Physiol 1970, 207:733-745.

30. Ghosh A, Sydekum E, Haiss F, Peduzzi S, Zorner B, Schneider R, Baltes C, Rudin M, Weber B, Schwab ME: Functional and Anatomical Reorganization of the Sensory-Motor Cortex after Incomplete Spinal Cord Injury in Adult Rats. J Neurosci 2009, 29:12210-12219.

31. Chakrabarty S, Shulman B, Martin JH: Activity-Dependent Codevelopment of the Corticospinal System and Target Interneurons in the Cervical Spinal Cord. J Neurosci 2009, 29:8816-8827.
32. Maier IC, Baumann K, Thallmair M, Weinmann O, Scholl J, Schwab ME: Constraint-Induced Movement Therapy in the Adult Rat after Unilateral Corticospinal Tract Injury. J Neurosci 2008, 28:9386-9403.

33. Frigon A, Yakovenko S, Gritsenko V, Tremblay ME, Barriere G: Strengthening Corticospinal Connections with Chronic Electrical Stimulation after Injury. J Neurosci 2008, 28:3262-3263.

34. Brosamle C, Schwab ME: Cells of origin, course, and termination patterns of the ventral, uncrossed component of the mature rat corticospinal tract. J Comp Neurol 1997, 386:293-303.

35. Luke R, Harris W, Bobet J, Sanelli L, Bennett DJ: Tail Muscles Become Slow but Fatigable in Chronic Sacral Spinal Rats With Spasticity. J Neurophysiol 2006, 95:1124-1133.

36. Landau WM, Bishop GH, Clare MH: Analysis of the form and distribution of cortical potentials under the influence of polarizing currents. $J$ Neurophysiol 1964, 27:788-813.

37. Gorman ALF: Differential patterns of activation of the pyramidal system elicited by surface anodal and cathodal cortical stimulation. $J$ Neurophysiol 1965, 29:547-64.

38. Terzoulo CA, Bullock TH: Measurement of imposed voltage gradient adequate to modulate neuronal firing. Proc Natl Acad Sci USA 1956 42:687-694.

39. Bindman $\sqcup$, Lippold OCJ, Redfearn JWT: Long-lasting changes in the level of the electrical activity of the cerebral cortex produced by polarizing currents. Nature 1962, 196:584-585.

40. Dan Y, Poo M: Spiking Timing-dependent plasticity: From synapse to perception. Physiol Rev 2006, 86:1033-1048.

41. Bliss TVP, Collingridge GL: A synaptic model of memory: Long-Term Potentiation in the hippocampus. Nature 1993, 361:31-39.

42. Landmesser LT, O'Donovan MJ: activation patterns of embryonic chick hindlimb muscles recorded in-ovo and in an isolated spinal cord preparation. J Physiol 1984, 347:189-204.

43. Katz LC, Shatz CJ: Synaptic activity and the construction of cortical circuits. Science 1996, 274:1133-1138.

44. Hanson MG, Landmesser LT: Normal patterns of spontaneous activity are required for correct motor axon guidance and the expression of specific guidance molecules. Neuron 2004, 46:687-701.

45. Hanson MG, Landmesser LT: Increasing the frequency of spontaneous rhythmic activity disrupts pool-specific axon fasciculation and pathfinding of embryonic spinal motoneurons. J Neurosci 2006, 26:12769-12780.

46. Gorassini M, Bennett DJ, Kiehn O, Eken T, Hultborn H: Activation patterns of hindlimb motor units in the awake rate and their relation to motoneuron intrinsic properties. J Neurophysiol 1999, 82:709-717.

47. Thomas CK, Ross BH: Distinct patterns of motor unit behavior during muscle spasms in spinal cord injured subjects. J Neurophysiol 1997, 77:2847-2850.

48. Harvey JP, Gorassini M, Bennett DJ: The spastic rat with sacral spinal cord injury. In Animal model of movement disorders. Edited by: Mark LeDoux. El Sevier Academic Press; 2005:691-697.

49. Hounsgaard J, Hultborn H, Jespersen B, Kiehn O: Bistability of alphamotoneurons in the decerebrate cat and in the acute spinal cat after intravenous 5-hydroxytryptophan. J Physiol 1988, 405:345-367.

50. Hsiao CF, Del Negro CA, Trueblood PR, Chandler SH: Ionic basis for serotonin-induced bistable membrane properties in guinea pig trigeminal motoneurons. J Neurophysiol 1998, 79:2847-2856.

51. Lee RH, Heckman CJ: Bistability in spinal motoneurons in vivo: systematic variations in persistent inward currents. J Neurophysiol 1998, 80:583-593.

doi:10.1186/1743-0003-7-46

Cite this article as: Ahmed: Dipolar cortico-muscular electrical stimulation: a novel method that enhances motor function in both normal and spinal cord injured mice. Journal of NeuroEngineering and Rehabilitation 2010 7:46. 